\title{
Anomalous small-angle $X$-ray scattering from charged soft matter
}

\author{
M. Sztucki ${ }^{\mathrm{a}}$, E. Di Cola, and T. Narayanan \\ European Synchrotron Radiation Facility, BP. 220, 38043 Grenoble, France
}

Received 16 January 2012 / Received in final form 23 March 2012

Published online 15 June 2012

\begin{abstract}
A review of recent applications of Anomalous Small-Angle X-ray Scattering (ASAXS) to charged soft matter systems is presented. Although the potential of ASAXS was realized in the eighties [1], applications to soft matter systems became feasible in recent years thanks to the technical developments at the synchrotron sources. Examples include both stiff chain and flexible polyelectrolytes, colloidal brush-like polyelectrolytes, DNA, RNA, and polysaccharides where the counterion profile could be determined with high precision and compared with theoretical models. In addition, ASAXS has also been found useful for microstructure characterization in soft materials. Finally, the present capability for ASAXS studies is illustrated by an example involving a surfactant micellar system.
\end{abstract}

\section{Introduction}

Anomalous Small-Angle X-ray Scattering (ASAXS) is a unique scattering method which allows limited contrast variation when the atomic adsorption edge of one of the constituent elements is at an accessible energy range. In a pioneering work, Stuhrmann $[1,2]$ presented the theoretical formalism of ASAXS and demonstrated the feasibility for biological macromolecular structural elucidation in solution. Nevertheless, for almost two decades, ASAXS was primarily restricted to the investigation of inorganic and metallic systems $[3,4]$. Quantitative ASAXS from soft matter and many biological materials was considered to be less feasible as the absorption edges of most relevant elements (carbon to calcium) are at rather low energies, causing important absorption effects and radiation damage of the sample. The relatively low concentration of ions present in soft matter systems results in only small changes in scattering intensity as a function of energy. Moreover, the intrinsic polydispersity of these systems further complicates the analysis.

However, quantitative ASAXS using high-brilliance synchrotron radiation offers a unique method for the structural characterization of charged soft matter systems. ASAXS allows to directly investigate the spatial distribution of free and bound counterions around a macroion with high precision by tuning the energy in the vicinity of the absorption edge of the counterions [5]. This information is not readily accessible by other techniques. In conventional scattering experiments, the contributions

\footnotetext{
${ }^{a}$ e-mail: sztucki@esrf.eu
} 
of the counterions and the macroions are usually superimposed and thus cannot easily be distinguished. In systems dominated by electrostatic interactions, counterions and ionic strength play crucial role in determining the microstructure and stability of the system [6]. Therefore, the precise knowledge of the spatial distribution of counterions is essential for the better understanding of charged soft matter systems like charge stabilized colloids, polyelectrolytes, proteins, surfactant micelles, membranes, etc. $[7,8]$.

This review briefly describes the principle of anomalous scattering and the experimental requirements for quantitative ASAXS measurements. Recent advances in instrumentation and data reduction have made ASAXS a suitable method for the quantitative characterization of the charge distribution in dilute and low contrast soft matter systems. This is demonstrated by means of selected ASAXS studies performed during the past decade. The state-of-the-art performance of the technique is illustrated using a cationic surfactant micellar system with bromide counterions.

\section{Anomalous small-angle $\mathrm{X}$-ray scattering (ASAXS)}

\subsection{Theory}

The potential of anomalous small-angle X-ray scattering was recognized in the eighties [2]. The technique makes use of the energy dependence of the X-ray scattering factor, $f$, of a particular element when the energy, $E$, of the incident X-ray beam is varied near the absorption edge of that element. This results in $f(E)$ being a complex function of energy:

$$
f(E)=f_{0}+f^{\prime}(E)+i f^{\prime \prime}(E)
$$

where the non-resonant term $f_{0}$ equals to the atomic number of the element, $f^{\prime}$ and $f^{\prime \prime}$ are the real and imaginary parts of $f . f^{\prime}$ and $f^{\prime \prime}$ are related through the KramersKronig relation. Tabulated values of $f^{\prime}$ and $f^{\prime \prime}$ as a function of energy are available for most elements [9]. For data analysis, it is necessary to convolute the tabulated values with the actual energy resolution $\Delta E / E$ of the instrument. In addition, $f^{\prime}$ and $f^{\prime \prime}$ could be also determined in-situ from the absorption coefficient [1].

Figure 1 presents the theoretical absorption edges of some elements in the energy range ( $7 \mathrm{keV}$ to $20 \mathrm{keV}$ ) which is most suitable for quantitative ASAXS. The accessible absorption edges range from iron to strontium K-edges and also include L-edges of some heavy elements (e.g. gold, platinum, lead, etc.). The absorption edges of the elements most relevant to soft matter and biological samples (carbon to calcium) are at lower energies, where quantitative SAXS is less feasible due to strong absorption and radiation damage. However, it is possible in some cases to replace sodium or potassium by rubidium, magnesium or calcium by strontium, sulphur by selenium, or chlorine by bromine without changing considerably the physical chemistry of the system.

For centrosymmetric particles, the energy dependent complex scattering factor, $f$, results in the radial scattering length density profile, $\rho$, of the resonantly scattering units itself becoming energy dependent $[3,10]$ :

$$
\rho(r, E)=\rho_{0}(r)+v(r) r_{e}\left[f^{\prime}(E)+i f^{\prime \prime}(E)\right]
$$

with $\rho_{0}(r)$ being the energy-independent scattering length density profile [11] (i.e. the usual contrast well below the absorption edge) and $v(r)$ being the spatial distribution of the anomalous scattering units (e.g. counterions); $r_{e}=2.818 \times 10^{-15} \mathrm{~m}$ is the classical electron radius. 


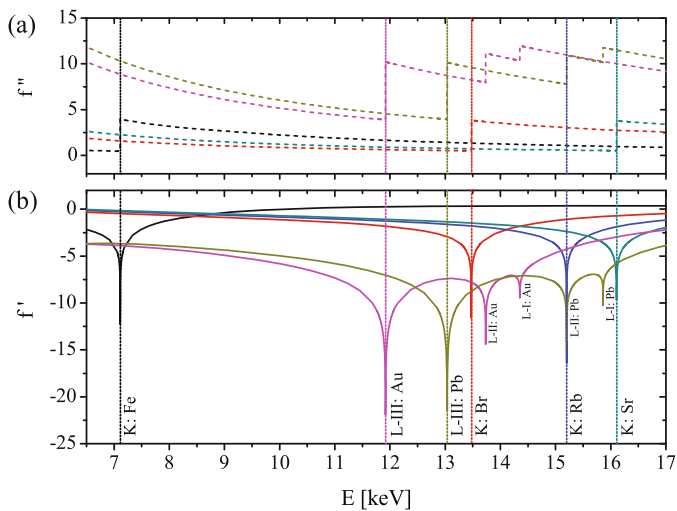

Fig. 1. Energy dependent real $f^{\prime}$ and imaginary $f^{\prime \prime}$ parts of the X-ray scattering factor $f$ of typical elements used in soft matter ASAXS depicting the anomalous effect [9]. The accessible absorption edges range from iron to strontium K-edges and also include L-edges of some heavy elements.

In the case of particulate systems, the scattered intensity, $I(q)$, as a function of the modulus of the scattering vector $(q)$ can be factorized as:

$$
I(q)=N F(q) F^{*}(q) S(q)
$$

with $N$ the number density of particles, $F(q)$ being the scattering amplitude given by the Fourier transform of $\rho(r, E), F^{*}(q)$ its complex conjugate and $S(q)$ the structure factor of interactions. Here $q=\frac{4 \pi}{\lambda} \sin \left(\frac{\theta}{2}\right)$, with $\theta$ the scattering angle and $\lambda$ the wavelength of the incident radiation. In the following, dilute, non-interacting systems are considered, where $S(q) \approx 1$ can be assumed. The total scattered intensity $I(q, E)$ becomes:

$$
I(q, E)=F_{0}^{2}(q)+2 f^{\prime}(E) F_{0}(q) v(q)+\left[f^{\prime 2}(E)+f^{\prime \prime 2}(E)\right] v^{2}(q)
$$

with $F_{0}(q)$ and $v(q)$ being the scattering amplitudes of 'normal' and anomalous scattering units, respectively.

The Eq. (4) contains three additive components: $F_{0}^{2}(q)$, the energy independent normal SAXS, $F_{0}(q) v(q)$, the cross-term involving the amplitudes of normal SAXS and the resonant scattering of the anomalous atoms, and $v^{2}(q)$, the weak resonant scattering term (self-term) originating from the anomalous atoms [5,12]. Having measured $I(q)$ at least at three energies, a decomposition of the scattered intensity into the three partial intensities in Eq. (4) is possible. The separation can be achieved by using the quadratic method proposed by Ballauff et al. [5] (see Sect. 3.1), neglecting the often very small contribution of $f^{\prime \prime}$ below the K-edges. Alternatively, a linear system of equations (constituted from measurements at $m \geq 3$ energies) can be represented by the following matrix equation and be solved numerically [11,13]:

$$
\left[\begin{array}{c}
I\left(q_{i}, E_{1}\right) \\
\vdots \\
I\left(q_{i}, E_{m}\right)
\end{array}\right]=\left[\begin{array}{ccc}
1 & 2 f^{\prime}\left(E_{1}\right) & f^{\prime 2}\left(E_{1}\right)+f^{\prime \prime 2}\left(E_{1}\right) \\
\vdots & \vdots & \vdots \\
1 & 2 f^{\prime}\left(E_{m}\right) & f^{\prime 2}\left(E_{m}\right)+f^{\prime \prime 2}\left(E_{m}\right)
\end{array}\right]\left[\begin{array}{c}
F_{0}^{2}\left(q_{i}\right) \\
F_{0}\left(q_{i}\right) v\left(q_{i}\right) \\
v^{2}\left(q_{i}\right)
\end{array}\right] .
$$

This approach offers higher accuracy as it fully takes into account $f(E)$. The use of the latter method is compulsory for energies, where the contribution of $f^{\prime \prime}$ cannot easily be neglected (i.e. at energies above the absorption edge or at L-edges). 
It should be also mentioned that in certain cases the simultaneous direct fitting of data sets recorded below and above the absorption edge provides valuable constraints in data modelling and helps to considerably improve the accuracy of the result (see Sect. 3.3 for more details).

\subsection{Experimental requirements}

The experimental requirements for ASAXS studies of soft matter systems are more demanding in terms of accuracy and repeatability [10] as compared to that in the case of hard materials [3]. This is mainly due to the relatively low concentration of anomalous species and the resulting small changes in $I(q)$ as a function of energy. Moreover, the sample stability and damage induced by the incident radiation brings additional constraints. Therefore, a proper surveillance of the recorded data quality is mandatory. The main demands on the setup can be grouped in four domains: (1) X-ray source and beamline optics, (2) 2D detectors and intensity monitors, (3) dedicated sample environment, and (4) data acquisition and online data reduction routines. In the following the main requirements for quantitative ASAXS measurements is summarized. A more detailed description of a specific setup at ID02 beamline, ESRF (Grenoble, France) can be found elsewhere [11].

The main requirements in terms of the X-ray source and beamline optics are high flux, positional stability of the beam, and low beam divergence. The beam has to be sufficiently monochromatised to provide a good energy resolution (energy bandwidth in the order of $\Delta E / E<0.02 \%$ ). Many relevant absorption edges can be reached in a spectral range spanning from $7 \mathrm{keV}$ to $20 \mathrm{keV}$ as discussed in Sect. 2.1. Concerning 2D detectors and intensity monitors, SAXS requires single photon sensitivity and high spatial resolution which can be fulfilled by state-of-the-art CCD-based X-ray detectors (e.g. the FReLoN camera using fibre-optically coupled Kodak CCD image sensor) [11]. Alternatively, energy-resolving pixel detectors (e.g. Pilatus detector by Dectris) can be used. However, even modern 2D pixel detectors cannot provide the required level of energy discrimination in order to avoid the fluorescence correction for measurements performed above the absorption edge [14]. Furthermore, a good intensity linearity and high stability of intensity monitors over the required energy range is essential. Note for example the coincidence of the K-edge of rubidium with the L-II-edge of lead (both $15.200 \mathrm{keV}$ ), which is an important constraint for the choice of the beamstop material. Detector efficiency, incident X-ray energy $(\Delta E>1 \mathrm{eV})$, incident flux, and sample transmission (accuracy better than $0.1 \%$ ) have to be carefully measured and calibrated. The sample transmission is best determined by a small intensity monitor directly incorporated in the primary beamstop which is placed in front of the detector.

For reliable ASAXS measurements with solutions, a dedicated sample environment is required, which allows the sequential recording of the sample, a standard to determine the absolute scattering intensity and a standard to calibrate the incident X-ray energy at each measurement. For example, a dedicated ASAXS flow-through capillary cell (with diameter of about $2 \mathrm{~mm}$ and wall thickness of $10 \mu \mathrm{m}$ ) has been developed for this purpose [11]. The scattered intensity from the sample and the corresponding solvent background can be measured at the same spot on the same capillary allowing very reliable subtraction of the background. For intensity calibration standard, water is recommended at higher $q$ ranges which allow precise measurement of water scattering $\left[I(q) \approx 1.6 \times 10^{-3} \mathrm{~mm}^{-1}\right.$ at $\left.25^{\circ} \mathrm{C}\right]$, while lupolen may be a better choice in the low $q$ region where the parasitic scattering is significantly greater than the water scattering. A solution containing known concentration of the anomalous atoms can be used as energy calibration standard (this also allows to detect any chemical shift of the absorption edge). To avoid radiation damage during an energy scan, provision 
should be made to refresh the sample by moving the solution in the flow-through cell using a motorized syringe.

ASAXS data have to be recorded at least at three energies around the absorption edge. In practice [11], it is more convenient to record ASAXS data of at least ten energies below and above the absorption edge. Energies should be chosen in a way that the values of $f^{\prime}$ are about equally spaced. Measurements at energies with equal $|f|$ below and above the absorption edge also permit an easy detection of fluorescence. Automatized online data reduction is essential to assure high quality of measurements. Typically, the two-dimensional SAXS patterns are corrected for detector artifacts, normalized by the solid angle subtended by the detector pixels and transmitted intensity, and azimuthally averaged to obtain the one dimensional profile. A specific ASAXS data reduction routine $[11,15,16]$ can now be used to correct for the energy dependence of the detector response and normalize the azimuthally integrated intensities to an absolute scale using the recorded scattering of the intensity calibration standard at each energy. A flat background due to fluorescence can be easily determined from the increase of scattering background at sufficiently high $q$ values (see Fig. 3 in Sect. 3.3). It has to be taken into account for measurements recorded above, but also slightly below the absorption edge. The latter is explained by the finite energy resolution of the incident X-rays $(\Delta E / E)$ which causes part of the energy spectrum to lie above the absorption energy in the close vicinity of the absorption edge [12]. Additional contribution could arise from resonant Raman scattering as the absorption edge is approached [17].

\section{Applications of ASAXS to soft matter}

In the pioneering ASAXS experiments, Stuhrmann analysed metal containing proteins such as haemoglobin, ferritin, and anomalous effect on the radius of gyration of DNA near the absorption edge of counterions $[1,2]$. In the case of the large subunit of ribosome $(1500 \mathrm{kD})$, measurements near phosphorous K-edge allowed separation of all three partial intensities [18]. Resonant scattering studies are also reported on freestanding liquid crystal films [19] and surfactant micellar solutions [3]. Nevertheless, the technique of contrast variation by anomalous scattering was mainly successful in the investigation of inorganic and metallic alloys for many years [4]. It is only in the last decade that the experimental accuracy has advanced enough to allow quantitative ASAXS experiments on soft matter.

\subsection{Polyelectrolytes}

In a series of publications, Ballauff and coworkers demonstrated that the developments at the modern synchrotron radiation sources could be exploited for ASAXS experiments on polyelectrolytes $[12,20-22]$. While the earlier experiments $[12,20]$ focused only on the analysis of the cross-term $F_{0}(q) v(q)$ (see Eq. (4)), the later works used the full information embodied in an ASAXS-experiment by deriving all three partial intensities, including the self-term $v^{2}(q)$ due to the resonant scattering of the counterions. Patel et al. [21] introduced a decomposition method for the evaluation of scattering curves measured at different energies below the absorption edge. They neglected the weak contribution of $f^{\prime \prime}$ (which is often true below the absorption edges) and expressed Eq. (4) as a quadratic function of $f^{\prime}$. Three partial intensities are then derived by linear regression from measurements recorded at different energies. This method is numerically more secure than simply subtracting two terms of larger intensities to derive the small resonant scattering term. The method was applied to 

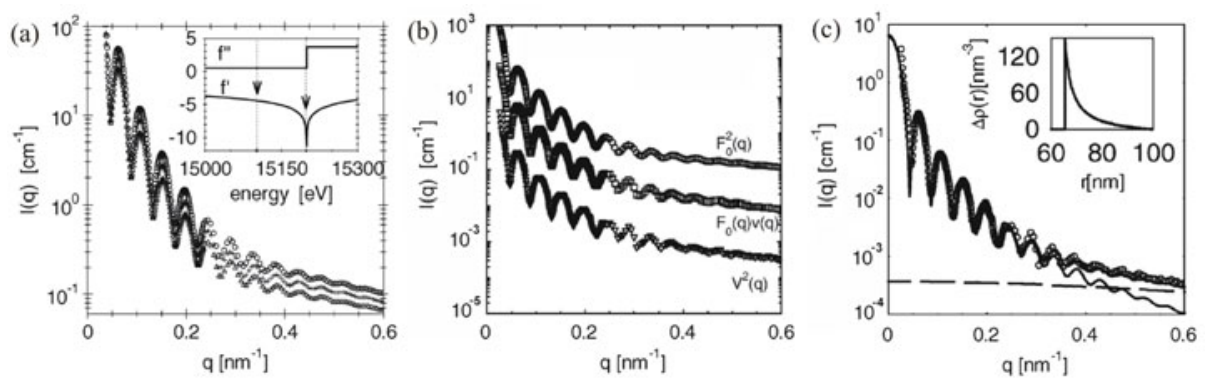

Fig. 2. (a) Dependence of the measured scattering intensity on the energy of the incident beam for the polyelectrolyte brushes with rubidium counterions [22]. Exemplary measurements corresponding to difference energies $-2737 \mathrm{eV}$ (circles), $-97.1 \mathrm{eV}$ (crosses), and $-1.1 \mathrm{eV}$ (triangles) below the K-edge of rubidium $(15200 \mathrm{eV})$. The inset shows the dispersion of rubidium. (b) Decomposition of the measured ASAXS intensities according to Eq. 4. (c) Partial scattering intensity of the counterions $v^{2}(q)$. The solid lines represent the fit by the radial profile of the electron density displayed in the inset. (taken from [22])

the study of rod-like polyelectrolytes consisting of a stiff poly(p-phenylene) backbone carrying positive charged groups which are balanced by bromide counterions. Partial intensities derived from measurements far below and in the vicinity of the bromine K-edge showed quantitative agreement with the predictions by the Poisson-Boltzmann cell model. An earlier experimental study testing the Poisson-Boltzmann model used the anomalous dispersion of iodine counterions [20], but suffered from larger statistical errors due to the limitations of ASAXS measurements at high energy of the absorption edge $(33 \mathrm{keV})$. Main experimental results and their quantitative comparison with Poisson-Boltzmann theory and computer simulations has been comprehensively reviewed by Ballauff and Jusufi [5].

Dingenouts et al. reported the first complete analysis by ASAXS of colloidal polyelectrolyte brushes [22]. The spherical polyelectrolyte brush was composed of a solid poly(styrene) core with a dense grafting of linear poly(acrylic acid) chains and rubidium counterions. ASAXS measurements were recorded at 13 different energies below the K-edge of rubidium $(15200 \mathrm{eV}$, see Fig. 2(a)). The three partial intensities (Fig. 2(b)) were derived by the method of linear regression as described before. The self-term $v^{2}(q)$ (see Fig. 2(c)) could be fitted by a radial profile that corresponded to highly stretched polyelectrolyte chains (density decaying as $r^{-2}$ ). The deduced spatial distribution of the counterions demonstrated that they are strongly correlated to the polyelectrolyte chains grafted to the surface of the core particles as predicted by theory [22]. Subsequent molecular dynamics simulations by Jusufi and Ballauff using a model of charged colloidal particles with counterions revealed distinct scattering features which depend on the strength of the electrostatic coupling between the macroion and counterions [23]. This can easily be distinguished by analyzing the partial intensities. In the case of strong coupling (i.e. the Bjerrum length much larger than the size of the counterion), the mean field approach such as the Poisson-Boltzmann theory is adequate to describe the cross-term and self-term of the counterions. For the weak coupling case (i.e. the Bjerrum length comparable to the size of the counterion), the strong counterion fluctuations make the fringes in the scattering function to smear out and the mean field description becomes inadequate for the self-term. In both cases, the cross-term contains the mean field part of the correlation between the counterions and macroions. Therefore, cross-term and self-term provide complementary information $[5]$. 
Goerigk et al. presented the first complete analysis of a flexible polyelectrolyte system [24]. They investigated the collapse process of sodium polyacrylate chains in aqueous solutions with the addition of strontium counterions. The pure-resonant scattering term was used to analyze the extent of $\mathrm{Sr}^{2+}$ condensation onto the polyacrylate coils $[24,25]$. A new methodology was proposed to determine the amount of cations localized around the collapsed chains with respect to the total amount of $\mathrm{Sr}$ ions in the solvent by analyzing the integral of the separated form factor of the counterions (so-called 'resonant invariant') [26]. In the collapsed state the resonant term vividly illustrated the pearl-necklace morphology consisting of collapsed sphere-like subdomains interconnected by stretched chain segments [24].

Contemporary to the work on synthetic polyelectrolytes, Das et al. [27] and Andresen et al. [28] demonstrated the ability of ASAXS to unravel the counterion atmosphere around DNA double helices. ASAXS allowed to validate the assumption that diffusively bound counterions of the same valence are interchangeable. They found good agreement between ASAXS results and calculations based on PoissonBoltzmann theory. As expected from the theory, the thickness of the counterion layer for monovalent ions was found to be about twice larger than for divalent ions. Using two absorption edges they were able to differentiate between the scattering profiles of monovalent $\left(\mathrm{Rb}^{+}\right)$and divalent $\left(\mathrm{Sr}^{2+}\right)$ ions competing in the charge neutralization of DNA [28]. The derived individual distribution functions were found to be invariant with reduced surface concentration summing to unity. However, a weak point in their study was that the analysis was based only on the cross-term and the self-term of the counterions was not determined. As noted before, the cross-term only reveals the mean correlation between counterions and the macroion [5]. In a subsequent related work, the charge screening efficiency and counterion distribution around short double stranded DNA and RNA molecules of comparable sequence was characterized [29]. The counterion mediated screening was found to be more efficient for RNA than DNA which has been attributed to the ability of RNA helix to draw monovalent and divalent ions closer. The experimental results once again agreed well with non-linear Poisson-Boltzmann model based calculations. For the same potential surface close to the nucleic acids, they found more 'bound' ions close to RNA than DNA. This distinction is relevant in understanding selective recognition of different types of short nucleic acid helices by target binding partners [29]. More recent work also reported a quantitative estimate of the number of counterions around DNA [30].

Similar to the studies on DNA and RNA, Horkay et al. [31] investigated the monovalent and divalent counterion distribution around hyaluronic acid, a copolymer of $\mathrm{N}$-acetyl-D-glucosamine and D-glucuronic acid which is a high molecular weight hydrophilic polysaccharide. They used rubidium and strontium as counterions to probe the different ion cloud around the biopolymer in the semidilute regime. They found good agreement with the Poisson-Boltzmann theory for the monovalent $\left(\mathrm{Rb}^{+}\right)$case. In monovalent-divalent ion mixtures, divalent counterions preferentially occupy the immediate vicinity of the macroion and size of the monovalent ion cloud shrinks with the addition of divalent counterions in contrast to the case of DNA where the distribution function of ions in competition was found to be invariant $[27,28]$.

In addition to synthetic and bio-polymers, ASAXS has been recently used to probe cation binding efficiency on to Keplerate polyoxometalate blackberry-like supermolecular structures [32]. This study confirmed that the less hydrated monovalent cations can bind stronger to a polyoxometalate macroion and reduce the critical salt concentration required for the formation of self-assembled colloidal structure.

In conventional ASAXS experiments, the fluorescence near and above the absorption edge is considered to be a nuisance. However, in phase separating systems an accurate fluorescence measurement could be used to quantify the partitioning of salt in the coexisting phases. Zhang et al. [33] used this approach to determine the salt 
concentration in a protein solution following liquid-liquid phase separation induced by the addition of multivalent counterions.

\subsection{Microstructure characterization}

ASAXS has found application in a wide variety of interdisciplinary fields involving soft materials. Braun et al. employed ASAXS near manganese K-edge for insitu characterization of the microstructure of the electrode material $\left(\mathrm{LiMn}_{2} \mathrm{O}_{4}\right)$ of a rechargeable battery cell [34]. Jokela et al., applied ASAXS to derive the size distribution of nanoparticles in palladium based catalysts on different polymer supports [35]. Soejima et al., investigated the structure of copper ion clusters in dehydrated N-isopropylacrylamide/sodium acrylate gel [36]. Their results indicated that copper clusters have the same size as sodium acrylate domains produced by microphase separation suggesting that the ion clusters are distributed in the hydrophilic sodium acrylate regions. In a composite material such as vulcanized rubber, Morfin et al. employed ASAXS to separate the scattering contribution arising from carbon black filler particles and $\mathrm{ZnO}$ particles inherited in the vulcanization process [37]. Sugiyama et al. investigated the distribution of copper ions in Nafion film such as that used in fuel cells and found that copper ions are preferentially located in a region where hydrophilic bases are accumulated [38]. Barnardo et al. studied the role of yttrium and zirconium ions during the sintering of sol-gel prepared yttria-stabilizedzirconia ceramics using in-situ double ASAXS across the yttrium and zirconium Kedges [13]. Their results revealed the dominant role of zirconia in the crystal nucleation and growth. Haas et al. probed even sub-nanometer scale patches of selenium on ruthenium nanoparticles used as catalysts for the oxygen reduction in membrane fuel cells [17].

Varga et al., exploited anomalous effects to locate bromine containing drug molecules (2,4-dibromophenol) along the phospholipid (DPPC) bilayer of liposomes [39]. A similar approach was used recently for the localization of epidermal growth factor receptor tyrosine kinase inhibitor drug in a PEGylated liposome (POPC) [40]. Bóta et al. [41] have used multilamellar vesicles as reactor of nanoparticles. ASAXS in this case allowed in-situ structural characterization of the cadmium sulfide and copper nanoparticles during their respective synthesis.

In other studies involving block copolymers, Lee et al. [42] performed contrast variation close to the gold $\mathrm{L}_{3}$-edge in order to reveal the morphology of bulk block copolymer/inorganic nanoparticle composites. They developed an analysis method to derive the partial scattering functions of the polymer and the nanoparticle phases which could be also suitable for other composite materials. In a more recent work, Sakou et al. [43] exploited ASAXS for probing the core-shell morphology of a block copolymer with bromostyrene as one of the blocks in a selective solvent. In this case, bromostyrene forms the core of the micelle and ASAXS near bromine K-edge allowed them to derive detailed information on the core-corona interface.

\subsection{Surfactant micellar system}

This section describes the state-of-the-art performance of the ASAXS technique using an optimized setup at the High-Brilliance SAXS beamline ID02 at the ESRF [11]. As an example, the investigation of a surfactant micellar system is presented where the counterion profile around the micelle can be deduced in a self-consistent manner $[11,14]$. The sample was composed of Tetradecyl-trimethylamonium bromide [TTAB, $\left.\mathrm{C}_{14} \mathrm{H}_{29} \mathrm{~N}\left(\mathrm{CH}_{3}\right)_{3} \mathrm{Br}\right]$ which is a well-known cationic surfactant, that in water forms 


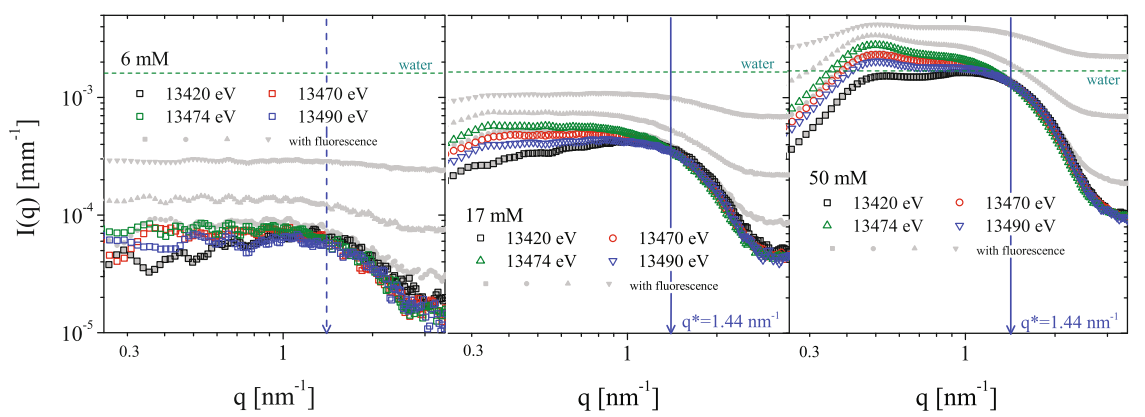

Fig. 3. ASAXS measurements of TTAB solutions at concentrations of $6 \mathrm{mM}, 17 \mathrm{mM}$, and $50 \mathrm{mM}$. Only few selected measurements recorded below and above the K-edge of bromine $(13474 \mathrm{eV})$ are shown for clarity. Open symbols correspond to data normalized to absolute intensity and corrected for fluorescence background. The closed grey symbols indicate the scattered intensity level before fluorescence subtraction. The scattering level of water is indicated to illustrate the limit of detection.

micelles above a critical micellar concentration (cmc) of about $3.6 \mathrm{mM}$ [44]. ASAXS measurements were performed at a sample to detector distance of $0.9 \mathrm{~m}$, using a single photon sensitive CCD detector (FReLoN). The following relative energies $(E-$ $13474 \mathrm{eV}$ ) near the bromine K-edge were used: $-1000 \mathrm{eV},-160 \mathrm{eV},-54 \mathrm{eV},-16 \mathrm{eV}$, $-10 \mathrm{eV},-6 \mathrm{eV},-4 \mathrm{eV},-2 \mathrm{eV}, \pm 0 \mathrm{eV},+2 \mathrm{eV},+4 \mathrm{eV},+8 \mathrm{eV},+16 \mathrm{eV},+46 \mathrm{eV}$ [11].

Figure 3 illustrates the limit of detection as compared to the scattering level of water for the three TTAB concentrations. The measurements (depicted with open symbols) show a pronounced change of the scattered intensity with energy which originates from the resonant scattering of the counterions at these concentrations, even at the lowest concentration of $6 \mathrm{mM}$. The figure also demonstrates the dominance of a flat background of bromine fluorescence (filled grey symbols) in the data recorded above, but also slightly below the absorption edge. This fluorescence background can be determined by the increase of scattering background at sufficiently high $q$ values $\left(3 \mathrm{~nm}^{-1}\right)$ and it can be subtracted provided that the counterion fluctuation effect is not significant.

Another remarkable feature evident in Fig. 3 is the existence of the so-called isoscattering point, i.e. a point where the scattered intensity is independent of the incident energy (or the scattering contrast of the counterion shell). This point is indicated in the figure at $q^{*}=1.44 \mathrm{~nm}^{-1}$ and is clearly visible in the case of the $50 \mathrm{mM}$ and $17 \mathrm{mM}$ sample. The limited statistics makes its detection, however, difficult in the case of the $6 \mathrm{mM}$ solution. The isoscattering point was first observed by Philipse et al. [45] while performing contrast variation experiments by adjusting temperature or composition of the solvent in light scattering experiments. Later on, the effect was also used for size determination of block copolymers and polymer latexes in SAXS measurements by tuning the scattering contrast of the solvent with the addition of glycerol or sucrose [46-48]. This technique allowed an accurate determination of the particle morphology without the necessity to explore the region of smallest $q$ values. For sufficiently monodisperse particles with spherical symmetry (e.g. coreshell particles), all scattering curves measured as a function of contrast cross each other at one point $q^{*}$ defined by [48]

$$
q^{*} R=\tan \left(q^{*} R\right)
$$

with $R$ being the mean radius of the particles. In the case of polydisperse systems only the first isoscattering point is visible and might even be smeared out at elevated polydispersities. In the case of TTAB micelles, a mean radius of about $R=3.1 \mathrm{~nm}$ can be estimated. 

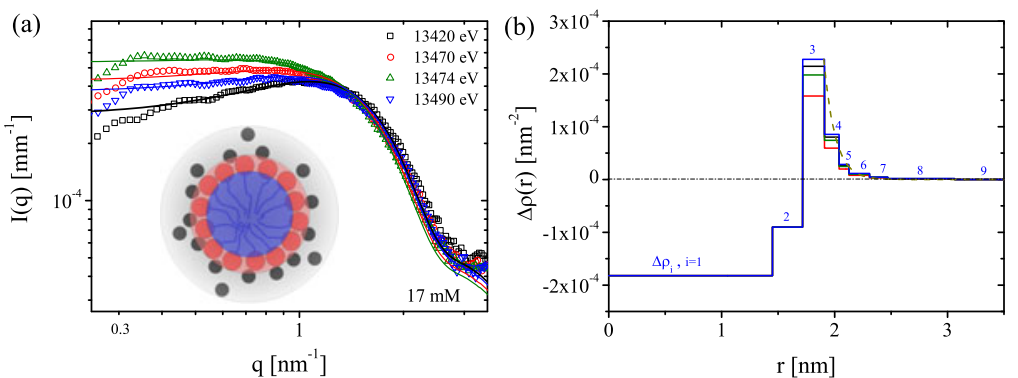

Fig. 4. (a) Selected datasets for $17 \mathrm{mM}$ TTAB solution together with their best fit model curves showing good agreement. A unique set of structural parameters for the core double shell model (inset) was used to fit data recorded at 14 different energies. (b) The optimum radial scattering length density profile derived from the fits. Only the scattering contrast within the counterion layer (second shell) changes according to the energy dependence of $f^{\prime}$ and $f^{\prime \prime}$. Counterions are concentrated in a layer of $0.34 \mathrm{~nm}$ thickness with an exponential decay up to $3.1 \mathrm{~nm}$ from the center of the core.

Quantitative analysis of the ASAXS measurements to determine the spatial distribution of the bromide counterions can be performed in two ways. A first approach is the separation of the measured intensities to partial intensities as described in section 2.1 [11]. The main focus of this section is to emphasize the advantages of a direct analysis of the ASAXS data which does not rely on any decomposition algorithm and simulates simultaneously all data measured at different energies (contrast variation). In the present case, this approach is recommended due to the pronounced anomalous effect observed. In addition, it has been shown that by directly simulating ASAXS curves below and above the absorption edge, the resolution of spatial distribution function of the counterions can be considerably refined as the full achievable range of f' and f" values are exploited [14]. The presented data analysis refers to the data recorded from the $17 \mathrm{mM}$ sample where the effect of particle interaction is not significant and $S(q) \approx 1$.

Figure 4 shows the simulation of the ASAXS measurements performed below and above the absorption edge. For clarity, only a limited number of curves are plotted, but fits for all 14 energies were simultaneously optimized. The micelles were simulated by a polydisperse core double-shell model [11]. The core represents the tetradecyl chains (blue sphere in the sketch in Fig. 4), the first shell the closely packed trimethylammonium head groups (red shell). The most interesting fit parameter is the spatial distribution of the bromide ions (black dots) within the outer shell (grey) approximated by six discrete levels (to allow analytic calculations). The scattering amplitude of the described core-shell model, $F_{c s}$, containing 8 concentric shells is given by

$$
F_{c s}\left(q, R_{1}\right) \propto \sum_{i=1}^{8}\left(\Delta \rho_{i}-\Delta \rho_{i+1}\right) V\left(R_{i}\right) F_{s}\left(q, R_{i}\right)
$$

where $F_{s}(q, R)=\frac{3[\sin (q R)-q R \cos (q R)]}{(q R)^{3}}$ is the well-known scattering amplitude of a sphere with radius $R$ and volume $V$ [49]. The scattering length density $\Delta \rho_{i}$ relative to the scattering length density of water $\left(\Delta \rho_{9}\right)$ is illustrated in Fig. $4(\mathrm{~b})$. For calculation of the scattered intensity $I(q)$, a Gaussian size distribution of the core (i.e. of $R_{1}$ ) around a mean value $R_{M 1}$ with variance $\sigma^{2}$ was used.

$$
I(q)=N \int_{0}^{\infty} \frac{1}{\sigma \sqrt{2 \pi}} \exp \left[-\frac{1}{2} \frac{\left(r-R_{M 1}\right)^{2}}{\sigma^{2}}\right] F_{c s}(q, r) F_{c s}^{*}(q, r) d r .
$$


For the best fit, a unique set of structural parameters (core radius, shell thickness, distribution function of counterions, contrast of core and first shell) was used. Only the scattering contrast within the second shell (counterion layer) changes according to the energy dependence of $f^{\prime}$ and $f^{\prime \prime}$ [see Fig. 4(b)]. An optimum fit (especially at smaller $q$ values) could only be achieved by adding an exponentially decaying tail to the counterion distribution function. Analyzing only the purely counterion contribution (self-term), this resolution was not achieved. The resulting structural parameters of the fit give a radius of $1.55 \mathrm{~nm}$ for the hydrophobic core (with $\sigma=0.36 \mathrm{~nm}$ ) and a thickness of $0.27 \mathrm{~nm}$ for the first shell representing the head groups. The distribution of the counterions is concentrated in a layer of $0.34 \mathrm{~nm}$ thickness (similar to the Stern layer) and decays exponentially to zero at a distance of $3.1 \mathrm{~nm}$ from the center of the core. This maximum size of the micelles (including the counterion layer) agrees well with the size determined from the analysis of the isoscattering point. The maximum number density of the ions is determined to be $3.8 \mathrm{~nm}^{-3}$, which is about $1 / 10$ of the closest possible packing of the ions. Nevertheless, about $72 \%$ of the bromide ions are directly bound to the surfactant head groups. This result is consistent with the previous estimates of counterion association from neutron scattering [50] and electrochemical methods [44]. It should be also mentioned that due to strong shape fluctuations of the micelles the assumption of a spherical core double-shell model is only an approximation and the system could be more realistically modelled by polydisperse ellipsoids. However, this mainly results in a more realistic value of the polydispersity as the orientational averaging of the ellipsoids also contributes to smear the form factor oscillations.

\section{Conclusion}

The examples presented in this review illustrate the potential of ASAXS for the structural characterization of charged soft matter systems. The use of high-brilliance synchrotron ASAXS offers a unique method to determine the spatial distribution of free and bound counterions around a macroion with high precision. In the case of polyelectrolytes, quantitative agreement of the results with theory and simulations has been achieved [5]. Recent instrumental developments made the technique even applicable to samples with low ion concentration. Two approaches for the data analysis were presented. The first analyzes - by numerical decomposition of normalized intensities - the three partial intensities of (1) normal SAXS, (2) the cross-term involving the amplitudes of normal SAXS and the resonant term of the counterions and (3) the resonant scattering due to counterions. An alternate approach is based on direct modelling of the measured intensities at several energies simultaneously (contrast variation). The former approach is suitable for weak anomalous effect, whereas direct modelling is more appropriate when the anomalous effect is more profound. The possibility of including data recorded both below and above the absorption edge provides a valuable constraint for refining the structural model. The fluorescence contribution detected close to and above the absorption edge can be well handled for many systems. Fluorescence measurement can even serve as a means for the accurate determination of the relative salt concentration within the sample.

ASAXS is applicable to a wide range of systems like polyelectrolytes, surfactant micelles, bilayer vesicles, DNA, RNA, polysaccharides and proteins. So far the examples involved relatively heavier counterions either intrinsically present or exchanged for lighter counterions such as sodium or potassium. Recent advance in SAXS near sulphur K-edge [51] is promising that quantitative ASAXS on soft matter systems could become feasible even at low energies involving more realistic lighter counterion. Another interesting aspect is the resonant enhancement of contrast which 
has been demonstrated in the case of polymer blend films [52]. This could be exploited to highlight weak nanostructural features in multicomponent systems. Of course, experiment at low energies is very challenging due to high absorption and consequently vanishing sample thickness, radiation damage, etc. Samples need to be either in the form of freestanding films or mounted in grazing incidence geometry.

The European Synchrotron Radiation Facility is acknowledged for the provision of beam time. M. Ballauff and S. Rosenfeldt are thanked for fruitful discussions.

\section{References}

1. H.B. Stuhrmann, Q. Rev. Biophys. 14, 433 (1981)

2. H.B. Stuhrmann, Adv. Polym. Sci. 67, 123 (1985)

3. A. Naudon, Modern Aspects of Small-Angle Scattering (Kluwer Academic Publishers, 1995), p. 203

4. M. Cianci, J.R. Helliwell, M. Helliwell, V. Kaucic, N.Z. Logar, G. Mali, N.N. Tusar, Cryst. Rev. 11, 245 (2005)

5. M. Ballauff, A. Jusufi, Coll. Pol. Sci. 284, 1303 (2006)

6. V.M. Prabhu, Curr. Opin. Colloid Interf. Sci. 10, 2 (2005)

7. C. Holm, P. Kekicheff, R. Podgornik, Electrostatic Effects in Soft Matter and Biophysics (Kluwer Academic Publishers, 2001)

8. D. Henderson, M. Holovko, A. Trokhymchuk, Ionic Soft Matter: Modern Trends in Theory and Applications (Kluwer Academic Publishers, 2005)

9. S. Brennan, P.L. Cowan, Rev. Sci. Instrum. 63, 850 (1992)

10. T. Narayanan, Lecture Notes in Physics (Non-crystalline diffraction) edited by T. Ezquerra, et al., 776 (Springer, Berlin-Heidelberg, 2009), p. 133

11. M. Sztucki, E. Di Cola, T. Narayanan, J. Appl. Cryst. 43, 1479 (2010)

12. N. Dingenouts, R. Merkle, X. Guo, T. Narayanan, G. Goerigk, M. Ballauff, J. Appl. Cryst. 36, 578 (2003)

13. T. Barnardo, K. Hoydalsvik, R. Winter, C.M. Martin, G.F. Clark, J. Phys. Chem. C 113, 10021 (2009)

14. M. Sztucki, E. Di Cola, T. Narayanan, J. Phys.: Conf. Ser. 272, 012004 (2011)

15. M. Sztucki, T. Narayanan, J. Appl. Cryst. 40, s459 (2007)

16. M. Sztucki, (2011) SAXSutilities download page, http://www.sztucki.de/SAXSutilities

17. S. Haas, G. Zehl, I. Dorbandt, I. Manke, P. Bogdanoff, S. Fiechter, A. Hoell, J. Phys. Chem. C 114, 22375 (2010)

18. H.B. Stuhrmann, J. Appl. Cryst. 40, s23 (2007)

19. P. Mach, R. Pindak, A.-M. Levelut, P. Barois, H. T. Nguyen, C.C. Huang, L. Furenlid, Phys. Rev. Lett. 81, 1015 (1998)

20. B. Guilleaume, J. Blaul, M. Ballauff, M. Wittemann, M. Rehahn, G. Goerigk, Eur. Phys. J. E 8, 299 (2002)

21. M. Patel, S. Rosenfeldt, M. Ballauff, N. Dingenouts, D. Pontoni, T. Narayanan, Phys. Chem. Chem. Phys. 6, 2962 (2004)

22. N. Dingenouts, M. Patel, S. Rosenfeldt, D. Pontoni, T. Narayanan, M. Ballauff, Macromolecules 37, 8152 (2004)

23. A. Jusufi, M. Ballauff, Macromol. Theory Simul. 15, 193 (2006)

24. G. Goerigk, R. Schweins, K. Huber, M. Ballauff, Europhys. Lett. 66, 331 (2004)

25. R. Schweins, G. Goerigk, K. Huber, Eur. Phys. J. E 21, 99 (2006)

26. G. Goerigk, K. Huber, R. Schweins, J. Chem. Phys. 127, 154908 (2007)

27. R. Das, T.T. Mills, L.W. Kwok, G.S. Maskel, I.S. Millett, S. Doniach, K.D. Finkelstein, D. Herschlag, L. Pollack, Phys. Rev. Lett. 90, 188103 (2003)

28. K. Andresen, R. Das, H.Y. Park, H. Smith, L.W. Kwok, J.S. Lamb, E.J. Kirkland, D. Herschlag, K.D. Finkelstein, L. Pollack, Phys. Rev. Lett. 93, 248103 (2004) 
29. S.A. Pabit, X. Qiu, J.S. Lamb, L. Li, S.P. Meisburger, L. Pollack, Nucleic Acids Res. 37, 3887 (2009)

30. S.A. Pabit, S.P. Meisburger, L. Li, J.M. Blose, C.D. Jones, L. Pollack, J. Am. Chem. Soc. 132, 16334 (2010)

31. F. Horkay, A.M. Hecht, C. Rochas, P.J. Basser, E. Geissler, J. Chem. Phys. 125, 234904 (2006)

32. J.M. Pigga, J.A. Teprovich Jr., R.A. Flowers, II, M.R. Antonio, T. Liu, Langmuir 26, $9449(2010)$

33. F. Zhang, R. Roth, M. Wolf, F. Roosen-Runge, M.W.A. Skoda, R.M.J. Jacobs, M. Sztucki, F. Schreiber (unpublished)

34. A. Braun, S. Seifert, P. Thiyagarajan, S.P. Cramer, E.J. Cairns, Electrochem. Comm. 3, 136 (2001)

35. K. Jokela, R. Serimaa, M. Torkkeli, V. Etelaniemi, K. Ekman, Chem. Mater. 14, 5069 (2002)

36. Y. Soejima, S. Kuwajima, M. Sugiyama, M. Annaka, A. Nakamura, N. Hiramatsu, K. Hara, J. Phys. Soc. Jpn. 72, 2110 (2003)

37. I. Morfin, F. Ehrburger-Dolle, I. Grillo, F. Livet, F. Bley, J. Synchrotron Rad. 13, 445 (2006)

38. M. Sugiyama, T. Mitsui, T. Sato, Y. Akai, Y. Soejima, H. Orihara, Y.-H. Na, K. Itoh, K. Mori, T. Fukunaga, J. Phys. Chem. B 111, 8663 (2007)

39. Z. Varga, A. Bota, G. Goerigk, J. Phys. Chem. B 110, 11029 (2006)

40. Z. Varga, S. Berenyi, B. Szokol, L. Orfi, G. Keri, I. Petak, A. Hoell, A. Bota, J. Phys. Chem. B 114, 6850 (2010)

41. A. Bóta, Z. Varga, G. Goerigk, J. Phys. Chem. B 111, 1911 (2007)

42. B. Lee, C.-T. Lo, S. Seifert, N.L.D. Rago, R.E. Winans, P. Thiyagarajan, Macromolecules 40, 4235 (2007)

43. M. Sakou, A. Takechi, M. Handa, Y. Shinohara, Y. Amemiya, H. Masunaga, H. Ogawa, N. Yagi, K. Sakurai, I. Akiba, J. Phys.: Conf. Ser. 272, 012022 (2011)

44. S.P. Moulik, M.E. Haque, P.K. Jana, A.R. Das, J. Phys. Chem. 100, 701 (1996)

45. A.P. Philipse, C. Smith, A.Vrij, J. Coll. Interf. Sci. 129, 335 (1988)

46. P. Hickl, M. Ballauff, A. Jada, Macromolecules 29, 4006 (1996)

47. M. Ballauff, Progr. Colloid Polym. Sci. 110, 76 (1998)

48. N. Dingenouts, J. Boltze, D. Pötschke, M. Ballauff, Adv. Polym. Sci. 144, 1 (1999)

49. J.S. Pedersen, Adv. Colloid Interface Sci. 70, 171 (1997)

50. N. Gorski, M. Gradzielski, H. Hoffmann, Langmuir 10, 2594603 (1994)

51. M. Handa, Y. Shinohara, H. Kishimoto, Y. Tamenori, N. Yagi, Y. Amemiya, J. Phys.: Conf. Ser. 272, 012014 (2011)

52. S. Swaraj, C. Wang, H. Yan, B. Watts, J. Luning, C.R. McNeill, H. Ade, Nano Lett. 10, 2863 (2010) 